\title{
ON LOCAL FINITE DIMENSIONAL APPROXIMATION OF $C^{*}$-ALGEBRAS
}

\author{
SORIN POPA
}

To Ed Effros, on the occasion of his 60th birthday.

\begin{abstract}
We prove a local finite dimensional approximation result for unital $\mathrm{C}^{*}$-algebras that are simple, have a trace, contain sufficiently many projections and are quasidiagonal.
\end{abstract}

\section{Introduction.}

Recall that a $C^{*}$-algebra $A$ is called quasidiagonal if it can be represented faithfully on a Hilbert space $\mathcal{H}$ such that there exist finite dimensional vector subspaces $\mathcal{H}_{i} \subset \mathcal{H}$, with $\mathcal{H}_{i} \nearrow \mathcal{H}$ and $\lim _{i}\left\|\left[\operatorname{proj}_{\mathcal{H}_{i}}, x\right]\right\|=0, \forall x \in A$ (see e.g., [V2]). By Voiculescu's theorem ([V1]), if $A$ is simple then this property doesn't in fact depend on the Hilbert space on which $A$ is represented, so that it can be reformulated in terms of the following local finite dimensional approximation property: A simple $C^{*}$-algebra is quasidiagonal if for any representation of $A$ on a Hilbert space $\mathcal{H}$, any finite set $F \subset A$ and any $\varepsilon>0$, there exists a finite dimensional vector subspace $0 \neq \mathcal{H}_{0} \subset \mathcal{H}$ such that $\left\|\left[\operatorname{proj}_{\mathcal{H}_{0}}, x\right]\right\|<\varepsilon, \forall x \in F$.

The result that we obtain in this paper gives an alternative, more intrinsic characterization of quasidiagonality, for unital simple $C^{*}$-algebras $A$ that have sufficiently many projections (abreviated s.m.p.), i.e., satisfy

(1.1) "There exists an abelian subalgebra $A_{0} \subset A$ such that for any $x=$ $x^{*} \in A$ and any $\varepsilon>0$, there exist a partition of $1_{A}$ with projections in $A_{0},\left\{p_{j}\right\}_{1 \leq j \leq n} \subset \mathcal{P}\left(A_{0}\right)$, and scalars $\left\{\alpha_{j}\right\}_{1 \leq j \leq n} \subset \mathbb{C}$, such that $\| p_{j} x p_{j}-$ $\alpha_{j} p_{j} \|<\varepsilon, 1 \leq j \leq n$."

Note that in case $A$ is separable this condition is easily seen to be implied by:

(1.1') "For any $x=x^{*} \in A$, any $p \in \mathcal{P}(A)$ and any $\varepsilon>0$, there exist a partition of $p$ with projections in $A,\left\{q_{i}\right\}_{1 \leq i \leq k} \subset \mathcal{P}(p A p)$, and scalars $\left\{\alpha_{i}\right\}_{1 \leq i \leq k} \subset \mathbb{C}$ such that $\left\|q_{i} x q_{i}-\alpha_{i} q_{i}\right\|<\varepsilon, 1 \leq i \leq k$." 
and this last property is clearly satisfied by $C^{*}$-algebras with real rank zero (see 2.2).

Thus we prove:

Theorem 1.2. Let $A$ be a unital, simple $C^{*}$-algebra with sufficiently many projections, in the sense of (1.1). Then $A$ is quasidiagonal if and only if it satisfies the following local finite dimensional approximation property: for any finite set $F \subset A$ and any $\varepsilon>0$, there exists a nonzero finite dimensional matrix subalgebra $Q \subset A$ with support $1_{Q}=s \in \mathcal{P}(A)$, such that:

(a) $\|[s, x]\|<\varepsilon, \forall x \in F$.

(b) $\operatorname{sxs} \underset{\varepsilon}{\in} Q, \forall x \in F$.

As we mentioned before, C-* algebras having real rank equal to zero (r.r.0) do have s.m.p. in the sense of (1.1). Yet r.r.0 is well known to be difficult to check, unless most of the structure of the C-* algebra is apriorically understood. In turn, property (1.1) seems to be easier to verify. In fact, large classes of algebras are seen to satisfy it just by their definition. For instance, if a C-* algebra is the crossed product of the continuous functions on a totally disconnected compact space $X$ by a group of automorphisms, with each one of the (non-trivial) automorphisms implementing a transformation without fixed points on $X$, then the $\mathrm{C}^{*}{ }^{*}$ algebra is easily seen to have s.m.p., by taking $A_{0}=\mathrm{C}(X)$. Also, Bellissard's quasi-crystal algebras ([B]) are shown to have s.m.p. in [BPo].

In fact, we will prove this type of local finite dimensional approximation for quasidiagonal $C^{*}$-algebras that satisfy even weaker conditions than (1.1) (see Definition 2.1), conditions that are checked, for instance, by $C^{*}$-algebras of the form $A=C(X) \rtimes \mathcal{G}$, where $X$ is totally disconnected and $\mathcal{G}$ a groupoid acting suitably on it. Many of the $C^{*}$-algebras in $[\mathbf{R}]$ have sufficiently many projections in this weaker sense.

The proof of 1.2 will be a straightforward addaptation to the $C^{*}$-algebra context of the proof to Connes' theorem in [Po1]. Thus, from quasidiagonality one has finite dimensional vector subspaces $\mathcal{H}_{0} \subset A\left(\hookrightarrow L^{2}(A\right.$, trace $\left.)\right)$ which are almost invariant (in the uniform norm) to given finite subsets $F \subset A$. By the same "local quantization" technique as in [Po1], one then gets projections $q \in A$ such that $q \mathcal{H}_{0}^{*} F \mathcal{H}_{0} q=\mathbb{C} q$ (approximately). Thus, $Q_{0}=\operatorname{sp} \mathcal{H}_{0} q \mathcal{H}_{0}^{*} \subset A$ will be close to an algebra $Q$, with the support $s$ almost invariant to $F$ and with $s F s$ almost contained in $Q$ (all the approximations being in the uniform norm).

Our initial motivation for this work was the problem, raised by Ed Effros in $[\mathbf{E}]$, of giving abstract, functional analytical characterizations to AF-algebras and to their subalgebras. While answering these problems seems still out 
of reach, we are hopeful that the above result and the method we use for proving it might be useful to their approach.

I thank E. Kirchberg, C. Pasnicu and G. Skandalis for comments. I am very grateful to G. Skandalis, J. Bellissard, G. Pisier, J.-L. Sauvageot and M. Enock for their warm hospitality and support, extended to me during my 1995-1996 stay at the Universities of Paris 6 and 7 and Paul Sabatier in Toulouse, where this work has been completed. I am most greatful to the Guggenheim Foundation for its financial support throughout this period.

\section{Local quantization.}

Let $A$ be a unital $C^{*}$-algebra and $\varphi: A \rightarrow \mathbb{C}$ a faithful state on $A$. Denote by $L^{2}(A, \varphi)$ the Hilbert norm $\|x\|_{\varphi}=\varphi\left(x^{*} x\right)^{1 / 2}, x \in A$, and by $\hat{x}$ the image of $x \in A$ as a vector in $L^{2}(A, \varphi)$. We identify $A$ with its image as the algebra of left multiplication operators on $L^{2}(A, \varphi)$. Thus we have $x \hat{y}=\widehat{x y}$ and $\varphi(x)=\langle x \hat{1}, \hat{1}\rangle, x, y \in A$.

Also, if $e_{0}$ denotes the one-dimensional orthogonal projection of $L^{2}(A, \varphi)$ onto $\mathbb{C} \hat{1}$ then $e_{0} x e_{0}=\varphi(x) e_{0}, x \in A$, and in fact, if $y \in A$ is so that $\|y\|_{\varphi}=\varphi\left(y^{*} y\right)^{1 / 2}=1$, then the orthogonal projection $p_{0}$ of $L^{2}(A, \varphi)$ onto $\mathbb{C} \hat{y}$ is given by $p_{0}=y e_{0} y^{*}$ and we have $p_{0} x p_{0}=\varphi\left(y^{*} x y\right) p_{0}$. More generally, if $\mathcal{H}_{0}=\sum_{j=1}^{n} \mathbb{C} \hat{y}_{j} \subset \hat{A}\left(\subset L^{2}(A, \varphi)\right)$ is a finite dimensional vector subspace, with $\varphi\left(y_{j}^{*} y_{i}\right)=\delta_{i j}$, and if $p_{0}$ denotes the orthogonal projection of $L^{2}(A, \varphi)$ onto $\mathcal{H}_{0}$, then $p_{0}=\sum_{j=1}^{n} y_{j} e_{0} y_{j}^{*}$ and

$$
p_{0} x p_{0}=\sum_{i, j=1}^{n} \varphi\left(y_{i}^{*} x y_{j}\right) y_{i} e_{0} y_{j}^{*}=\sum_{i, j=1}^{n} \varphi\left(y_{i}^{*} x y_{j}\right) e_{i j}^{\prime} \text {, }
$$

with $e_{i j}^{\prime}=y_{i} e_{0} y_{j}^{*}$ being a matrix unit for the algebra

$$
\sum_{i, j} \mathbb{C} y_{i} e_{0} y_{j}^{*}=p_{0} \mathcal{B}\left(L^{2}(A, \varphi)\right) p_{0}=\mathcal{B}\left(\mathcal{H}_{0}\right) \cong M_{n \times n}(\mathbb{C}) .
$$

In this section we construct projections $q$ in the algebra $A$ which approximately behave like $e_{0}$ on finite sets of elements of $A$. We will generically call such a construction "local quantization."

We restrict ourselves to the case when $\varphi$ is a trace, $\varphi=\tau$, a situation in which the norm \|\|$_{\varphi}$ will simply be denoted \|\|$_{2}$.

To do the local quantization we will need the algebra $A$ to have sufficiently many projections, like for instance in (1.1). We can in fact allow a greater generality. 
Definition 2.1. Let $A$ be a unital $C^{*}$-algebra with a faithful trace $\tau$. We say that $A$ has $\tau$-sufficiently many projections ( $\tau$-s.m.p.) if for any separable subset $B \subset A$ and any $\varepsilon>0$, there exists a partition of the identity with projections in $A,\left\{p_{j}\right\}_{1 \leq j \leq n} \subset \mathcal{P}(A)$, with $\tau\left(p_{j}\right)<\varepsilon, \forall j$, such that for any finite subset $F \subset B$ and any $\delta>0$, there exist mutually orthogonal projections $\left\{q_{i j}\right\}_{1 \leq i \leq m_{j}}$ under $p_{j}, 1 \leq j \leq n$, and scalars $\alpha_{i j}^{x} \in \mathbb{C}, 1 \leq i \leq m_{j}$, $1 \leq j \leq n, x \in F$, such that

$$
\begin{gathered}
\left\|q_{i j} x q_{i j}-\alpha_{i j}^{x} q_{i j}\right\|<\delta, \quad \forall i, j, \quad \forall x \in F \\
\tau\left(p_{j}-\sum_{i} q_{i j}\right)<\delta \tau\left(p_{j}\right), \quad \forall j .
\end{gathered}
$$

Note that for a unital $C^{*}$-algebra $A$ with a faithful trace $\tau$ for which $A^{\prime \prime} \subset$ $\mathcal{B}\left(L^{2}(A, \tau)\right)$ has no minimal projections (e.g., when $A$ is infinite dimensional and simple and $\tau$ is factorial), 2.1 is clearly implied by the following property:

(2.1') "There exists an abelian subalgebra $A_{0} \subset A$ such that $\forall x=x^{*} \in A$, $\forall \varepsilon>0, \exists\left\{p_{j}\right\}_{0 \leq j \leq n} \subset \mathcal{P}\left(A_{0}\right)$, partition of $1_{A}$, and scalars $\left\{\alpha_{j}\right\}_{1 \leq j \leq n} \subset$ $\mathbb{C}$ such that:

$$
\begin{aligned}
\left\|p_{j} x p_{j}-\alpha_{j} p_{j}\right\| & <\varepsilon, \text { for } 1 \leq j \leq n, \\
\tau\left(p_{0}\right) & <\varepsilon . "
\end{aligned}
$$

This condition is in turn obviously implied by (1.1). In the same spirit, let us also mention a weak version of condition $\left(1.1^{\prime}\right)$, which in case $A$ is separable implies (2.1).

$\left(2.1^{\prime \prime}\right)$ "For any $x=x * \in A$ any $p \in \mathcal{P}(A)$ and any $\varepsilon>0$, there exists a partition of $p$ with projections in $A,\left\{q_{j}\right\}_{0 \leq j \leq n}$, and acalars $\left\{\alpha_{j}\right\}_{1 \leq j \leq n}$, such that:

$$
\begin{aligned}
\left\|q_{j} x q_{j}-\alpha_{j} q_{j}\right\| & <\varepsilon, \text { for } 1 \leq j \leq n, \\
\tau\left(q_{0}\right) & <\varepsilon . "
\end{aligned}
$$

The above condition is of course implied by $\left(1.1^{\prime}\right)$. Altogether we have:

Proposition 2.2. Let $A$ be a unital $C^{*}$-algebra:

$1^{\circ}$. If $A$ has real rank zero $([\mathbf{B r P}])$, i.e., $\forall x=x^{*} \in A, \forall \varepsilon>0$, $\exists a=a^{*} \in A$, a with finite spectrum, such that $\|x-a\|<\varepsilon$, then $A$ satisfies condition $\left(1.1^{\prime}\right)$.

$2^{\circ}$. If $A$ is separable and satisfies $\left(1.1^{\prime}\right)$ (respectively $\left(2.1^{\prime \prime}\right)$ ) then it satisfies (1.1) (respectively $\left(2.1^{\prime}\right)$ ) too. 
$3^{\circ}$. If A satisfies (1.1) (resp. (1.1')) then it satisfies (2.1') (resp. (2.1")) for any faithful trace $\tau$.

$4^{\circ}$. If $A$ has a faithful trace $\tau$ for which $\left(2.1^{\prime}\right)$ is satisfied and so that $A^{\prime \prime} \subset \mathcal{B}\left(L^{2}(A, \tau)\right)$ has no minimal projections, then $A$ has $\tau$-s.m.p. (i.e., satisfies 2.1$)$.

$5^{\circ}$. If A satisfies (1.1') then it has $\tau$-s.m.p. for any faithful trace $\tau$ for which $A^{\prime \prime}$ has no minimal projections. (NB: $A$ is not assumed separable!).

$6^{\circ}$. Assume $A$ has a faithful trace $\tau$ and an abelian $C^{*}$-subalgebra $A_{0} \subset A$ generated by projections, with the property that the set of partial isometries $u$ in $A$ which normalize $A_{0}$, i.e., $u A_{0} u^{*}=u u^{*} A_{0}, u u^{*} \in A_{0}$, generate $A$ as a $C$-* algebra. Assume also that for each such normalizing $u$ the $\tau$-measure of the boundary of $X_{u}$ in $X$ is equal to zero, where $X_{u}$ denotes the subset of $X$ (= spectrum of $\left.A_{0}\right)$ fixed by the homeomorphism on (part of) $X$ implemented by $u$. Then $A$ satisfies $\left(2,1^{\prime}\right)$. Thus, if in addition $A^{\prime \prime}$ has no minimal projections, then $A$ has $\tau$-s.m.p.

Proof. $3^{\circ}$ is trivial.

To prove $4^{\circ}$ note that if $\left(2.1^{\prime}\right)$ is satisfied then $A_{0}^{\prime \prime}$ is maximal abelian in $A^{\prime \prime}$ and the projections in $A_{0}$ are dense in the norm \|\|$_{2}$ in the set of projections of $A_{0}^{\prime \prime}$. This implies that one can take the projections $p_{j} \in A_{0}$ in ' $\left(2.1^{\prime}\right)$ to have as small trace as wanted.

$1^{\circ}$ is an imediate consequence of the fact that $A$ has real rank zero implies $p A p$ has real rank zero for any projection $p$ in $A$.

If $A$ is separable and satisfies (1.1') then let $\left\{x_{n}\right\}_{n} \subset A$ be a sequence of self adjoint elements norm dense in $A$. By $\left(1.1^{\prime}\right)$ one can construct recursively finite dimensional abelian ${ }^{*}$-subalgebras $\mathbb{C} 1=A_{1}^{0} \subset A_{2}^{0} \subset \cdots$, such that $E_{A_{n}^{0} \cap \cap A}\left(x_{n}\right) \underset{1 / n}{\in} A_{n}^{0}$. Then $A_{0} \stackrel{\text { def }}{=} \overline{\bigcup A_{n}^{0}} \subset A$ will satisfy (1.1), thus proving $2^{\circ}$.

The proof of $5^{\circ}$ is similar to the proof of $2^{\circ}$.

The proof of $6^{\circ}$ is an easy exercise.

From the above, we thus have large classes of algebras with "many projections" in the sense we need, as algebras with real rank zero and many crossed product algebras $C(X) \rtimes \mathcal{G}$, with $X$ a totally disconnected compact set and $\mathcal{G}$ a groupoid acting on it (e.g., like in [R]), satisfy (2.1) (cf. 2.2.6 ${ }^{\circ}$ ). Also, the quasi-crystal algebras in $[\mathbf{B}]$ are shown in $[\mathbf{B P o}]$ to be simple, have unique trace, satisfy the Dixmier property and have s.m.p. in the sense of (1.1), although it is not known whether they have r.r.0 or not.

Theorem 2.3. Let $A$ be a unital, simple, infinite dimensional $C^{*}$-algebra with a factorial trace $\tau$. If $A$ has $\tau$-s.m.p., i.e., satisfies 2.1, then for any finite set $F \subset A$ and any $\varepsilon>0$, there exists a partition of $1_{A}$ with projections 
$\left\{q_{i}\right\}_{0 \leq i \leq m} \subset \mathcal{P}(A)$ such that:

$$
\begin{gathered}
\left\|q_{i} x q_{i}-\tau(x) q_{i}\right\|<\varepsilon, \forall x \in F, 1 \leq i \leq m, \\
\tau\left(q_{0}\right)<\varepsilon .
\end{gathered}
$$

Proof. Let $M$ be the von Neumann algebra generated by $A \subset \mathcal{B}\left(L^{2}(A, \tau)\right)$. Thus, $M$ is a type $\mathrm{II}_{1}$ factor, $\hat{1}$ is a trace vector for $M$ and $L^{2}(A, \tau)=$ $L^{2}(M, \tau)$. By Property 2.1 and a standard separability argument, it follows that we may suppose $A$ is separable in the uniform norm, and thus $M$ is separable in the norm \|\|$_{2}$.

We may clearly suppose the finite set $F \subset A$ is so that $x=x^{*},\|x\| \leq 1$, $\tau(x)=0, \forall x \in F$. With this assumption, note that if $x \in F, x \neq 0$, then $s\left(x_{+}\right) \neq 1, s\left(x_{-}\right) \neq 1$, (where for $a=a^{*} \in M^{\omega}, s(a)$ denotes the support projection of $a$ ). Denote $\alpha=\max \left\{\tau\left(s\left(x_{ \pm}\right)\right), x \in F, x \neq 0\right\}$ (so $\alpha<1)$. Let $\left\{p_{j}\right\}_{1 \leq j \leq n}$ be a partition of the identity in $\mathcal{P}(A)$ such that $\tau\left(p_{j}\right)<\min \left\{\varepsilon^{2} / 4^{2} \cdot 8^{2},(1-\alpha) / 2\right\}, \forall j \leq n$. Let $\omega$ be a free ultrafilter on $\mathbb{N}$ and $M^{\omega}$ be the ultrapower algebra associated to $M$ and $\omega$. By [Po2] there exists a unitary element $u=\left(u_{n}\right)_{n} \in M^{\omega}$ such that $u M u^{*}$ is free with respect to $M$ (so that in particular $u p_{j} u^{*}$ are free with respect to $M$ ). By the density of $A$ in $M$ we may assume $u_{n} \in \mathcal{U}(A), \forall n$. By [V3] it follows that if $x$ is of the form $f-\tau(f) 1$, with $\tau(f)+\tau\left(p_{j}\right) \leq 1 \forall j$, then $\left\|u p_{j} u^{*} x u p_{j} u^{*}\right\|<\varepsilon / 4$. In general we have $x_{ \pm} \leq 1$ so $x_{+}=\sum_{n=1}^{\infty} 2^{-n} f_{n}^{+}$, $x_{-}=\sum_{n=1}^{\infty} 2^{-n} f_{n}^{-}$, for some projections $f_{n}^{ \pm}$with $\tau\left(f_{n}^{ \pm}\right) \leq \alpha, \forall n$. Since $\sum_{n} 2^{-n} \tau\left(f_{n}^{+}\right)=\tau\left(x_{+}\right)=\tau\left(x_{-}\right)=\sum_{n} 2^{-n} \tau\left(f_{n}^{-}\right)$, we get

$$
x=x_{+}-x_{-}=\sum_{n=1}^{\infty} 2^{-n}\left(f_{n}^{+}-\tau\left(f_{n}^{+}\right) 1\right)-\sum_{n=1}^{\infty} 2^{-n}\left(f_{n}^{-}-\tau\left(f_{n}^{-}\right) 1\right)
$$

and so we have the estimate:

$$
\begin{aligned}
\left\|u p_{j} u^{*} \operatorname{xup}_{j} u^{*}\right\| \leq \sum_{n=1}^{\infty} 2^{-n}\left(\| u p_{j} u^{*}\right. & \left(f_{n}^{+}-\tau\left(f_{n}^{+}\right) 1\right) u p_{j} u^{*} \| \\
& \left.+\left\|u p_{j} u^{*}\left(f_{n}^{-}-\tau\left(f_{n}^{-}\right) 1\right) u p_{j} u^{*}\right\|\right)<\varepsilon / 2 .
\end{aligned}
$$

By Property (2.1), for each $n$ there exist mutually orthogonal projections $\left\{q_{i}^{n}\right\}_{1 \leq i \leq k_{n}} \subset \mathcal{P}(A)$, with each $q_{i}^{n}$ being under some $p_{j}$, and scalars $\alpha_{i, x}^{n} \in \mathbb{C}$, $1 \leq i \leq k_{n}, x \in F$, such that:

$$
\lim _{n \rightarrow \infty}\left(\sup _{i, x}\left\{\left\|q_{i}^{n} u_{n}^{*} x u_{n} q_{i}^{n}-\alpha_{i, x}^{n} q_{i}^{n}\right\| \mid 1 \leq i \leq k_{n}, x \in F\right\}\right)=0
$$




$$
\lim _{n \rightarrow \infty} \tau\left(1-\sum_{i} q_{i}^{n}\right)=0 .
$$

But for each $n$ the application

$$
q^{n} M q^{n} \ni y \mapsto \sum_{i} q_{i}^{n} y q_{i}^{n} \in q^{n} M q^{n}
$$

where $q^{n}=\sum_{i} q_{i}^{n}$, defines a trace preserving conditional expectation $E_{n}$. Since $\left(q^{n}\right)_{n}=1$ in $M^{\omega}$, it follows that:

$$
M^{\omega}=\prod_{n \rightarrow \omega}\left(q^{n} M q^{n}\right) \ni\left(q^{n} y_{n} q^{n}\right)_{n} \mapsto\left(E_{n}\left(q^{n} y_{n} q^{n}\right)\right)_{n}=\left(\sum_{i} q_{i}^{n} y_{n} q_{i}^{n}\right)_{n} \in M^{\omega}
$$

defines a trace preserving conditional expectation $E$ as well. Thus, we have for $x \in F$ the estimates

$$
\left\|E\left(\left(q^{n} u_{n}^{*} x u_{n} q^{n}\right)_{n}\right)\right\| \leq\left\|\left(q^{n}\left(\sum_{j} p_{j} u_{n}^{*} x u_{n} p_{j}\right) q^{n}\right)\right\|<\varepsilon / 2
$$

where in $M^{\omega}$ we have for $x \in F$ :

$$
E\left(\left(q^{n} u_{n}^{*} x u_{n} q^{n}\right)_{n}\right)=\left(\sum_{i} \alpha_{i, x}^{n} q_{i}^{n}\right)_{n} .
$$

From the above norm inequalities, it follows that for any $\delta>0$, there exists a sufficiently large $n$ such that the spectral projection $e_{n}^{x}$ of $\left|\sum_{i} \alpha_{i, x}^{n} q^{n}\right|=$ $\sum_{i}\left|\alpha_{i, x}^{n}\right| q_{i}^{n}$ corresponding to the interval $(0, \varepsilon / 2+\delta)$ satisfies

$$
\tau\left(e_{n}^{x}\right) \geq 1-\delta, \quad \forall x \in F .
$$

Thus, if $e=\bigwedge_{x \in F} e_{n}^{x}$ then $\tau(e) \geq 1-|F| \delta$, so that if we denote $q_{i}^{\prime}=q_{i}^{n} e$, $1 \leq i \leq m=k_{n}$, then we have:

$$
\begin{gathered}
\left\|q_{i}^{\prime} u_{n}^{*} x u_{n} q_{i}^{\prime}\right\|<\varepsilon / 2+\delta, \quad \forall x \in F, 1 \leq q_{i} \leq m \\
\tau\left(1-\sum_{i} q_{i}^{\prime}\right)<|F| \delta+\delta .
\end{gathered}
$$

Thus, if $\delta>0$ is chosen so that $\delta<\varepsilon /(|F|+1), \delta<\varepsilon / 2$, and we put $q_{i} \stackrel{\text { def }}{=} u_{n} q_{i}^{\prime} u_{n}^{*}, 1 \leq i \leq m$ and $q_{0} \stackrel{\text { def }}{=} 1-\sum_{1 \leq i \leq m} q_{i}$, then we have:

$$
\left\|q_{i} x q_{i}\right\|<\varepsilon, \forall x \in F, \quad 1 \leq i \leq m,
$$




$$
\tau\left(q_{0}\right)<\varepsilon
$$

We can now deduce a version of 2.3 which shows more clearly that, indeed, one can "simulate" the action of the one-dimensional projection $e_{0}$ on finite sets of elements and finite dimensional vector spaces by projections in $A$.

Corollary 2.4 (Local quantization). Let $A$ be a unital, simple $C^{*}$ algebra with a factorial trace $\tau$ and $\tau$-s.m.p. If $\mathcal{H}_{0}=\sum_{j=1}^{n} \mathbb{C} y_{j} \subset A$ is a finite dimensional vector subspace, with $\tau\left(y_{j}^{*} y_{i}\right)=\delta_{i j}, 1 \leq i, j \leq n$, and $F \subset A$ is a finite set, then for any $\varepsilon>0$ there exist mutually orthogonal projections $\left\{q_{i}\right\}_{1 \leq i \leq m}$ in $A$ and partial isometries $\left\{v_{i k}\right\}_{\substack{1 \leq i \leq m \\ 1 \leq k \leq n}}$ in $A$ such that if we denote $e_{i j}^{k}=v_{i k} v_{j k}^{*}, 1 \leq i, j \leq n, 1 \leq k \leq m$, and $s_{k}=\sum_{i} e_{i i}^{k}, 1 \leq k \leq m$, then we have

$1^{\circ} . v_{j k}^{*} v_{i k}=\delta_{i j} q_{k}, 1 \leq i, j \leq n, 1 \leq k \leq m$ (thus, $\forall k,\left\{e_{i j}^{k}\right\}_{1 \leq i, j \leq n}$ is a matrix unit for an $n \times n$ matrix algebra $Q_{k}$ ).

$2^{\circ} .\left\|v_{j k}-y_{j} q_{k}\right\|<\varepsilon, \forall j, k$.

$3^{\circ} .\left\|s_{k} x s_{k}-\sum_{i, j=1}^{n} \tau\left(y_{i}^{*} x y_{j}\right) e_{i j}^{k}\right\|<\varepsilon, \forall x \in F, \forall 1 \leq k \leq m$.

$4^{\circ} \cdot \tau\left(1-\sum_{k=1}^{m} q_{k}\right)<\varepsilon,\left\|\sum_{j=1}^{n} y_{j} y_{j}^{*}-\sum_{k=1}^{m} s_{k}\right\|_{1}<\varepsilon$.

Moreover, if the left supports of $y_{j}$ are all under a projection $e \in A$, i.e., $e y_{j}=y_{j}, \forall j$, then the partial isometries $v_{j k}$ can be chosen such that $e v_{j k}=v_{j k}, \forall j, k$, so in particular $e_{i j}^{k}$ are in $e A e, \forall i, j, k$.

Proof. Let $\delta>0$. We can apply 2.3 to the set $F^{\prime}=\left\{y_{j}^{*} x y_{i} \mid 1 \leq i, j \leq n, x \in\right.$ $F \cup\{1\}\}$ to get mutually orthogonal projections $\left\{q_{j}\right\}_{1 \leq j \leq m}$ such that

$$
\begin{gathered}
\left\|q_{k} y_{i}^{*} x y_{j} q_{k}-\tau\left(y_{i}^{*} x y_{j}\right) q_{k}\right\|<\delta, \quad 1 \leq i, j \leq n, 1 \leq k \leq m, x \in F \cup\{1\} \\
\tau\left(1-\sum_{k} q_{k}\right)<\delta .
\end{gathered}
$$

A standard perturbation argument (see, e.g., [Di]) then shows that there exist partial isometries $v_{i k} \in A, 1 \leq i \leq n, 1 \leq k \leq m$, such that

$$
\begin{gathered}
v_{i k}^{*} v_{j k}=\delta_{i j} q_{k} \\
\left\|v_{j k}-y_{j} q_{k}\right\|<12 n^{2} \delta^{1 / 2^{n}} \stackrel{\text { def }}{=} f(\delta) .
\end{gathered}
$$


By applying the triangle inequality, from the above estimates we deduce:

$$
\begin{aligned}
\left\|v_{j k}^{*} x v_{i k}-q_{k} y_{j}^{*} x y_{i} q_{k}\right\| & <2 f(\delta)(1+\delta) \\
\left\|v_{j k}^{*} x v_{i k}-\tau\left(y_{j}^{*} x y_{i}\right) q_{k}\right\| & <2 f(\delta)(1+\delta)+\delta=f^{\prime}(\delta) .
\end{aligned}
$$

By letting $e_{i j}^{k}=v_{i k} v_{j k}^{*}$ and $s_{k}=\sum_{i} e_{i i}^{k}$, this finally yields:

$$
\begin{aligned}
\left\|s_{k} x s_{k}-\sum_{i, j} \tau\left(y_{i}^{*} x y_{j}\right) e_{i j}\right\| & \leq \sum_{i, j}\left\|v_{i k} v_{i k}^{*} x v_{j k} v_{j k}^{*}-\tau\left(y_{i}^{*} x y_{j}\right) v_{i k} v_{j k}^{*}\right\| \\
& =\sum_{i, j}\left\|v_{i k}^{*} x v_{j k}-\tau\left(y_{i}^{*} x y_{j}\right) q_{k}\right\| \\
& \leq n^{2} f^{\prime}(\delta) .
\end{aligned}
$$

Similarily we have:

$$
\left\|\sum_{j} y_{j} q_{k} y_{j}^{*}-s_{k}\right\|<n f^{\prime}(\delta)
$$

for all $1 \leq k \leq m$, so that we get:

$$
\begin{aligned}
& \left\|\sum_{j} y_{j} y_{j}^{*}-\sum_{k} s_{k}\right\|_{1} \\
& \leq \tau\left(\sum_{j} y_{j}\left(1-\sum_{k} q_{k}\right) y_{j}^{*}\right)+\sum_{k}\left\|\sum_{j} y_{j} q_{k} y_{j}^{*}-s_{k}\right\|_{1} \\
& <\tau\left(1-\sum_{k} q_{k}\right)\left\|\sum_{j} y_{j}^{*} y_{j}\right\|+\sum_{k}\left(n f^{\prime}(\delta)\right) \tau\left(\sum_{j} y_{j} q_{k} y_{j}^{*}\right) \\
& \leq \delta\left\|\sum_{j} y_{j}^{*} y_{j}\right\|+n f^{\prime}(\delta) \sum_{j} \tau\left(y_{j} y_{j}^{*}\right) .
\end{aligned}
$$

Thus, if $\delta$ is chosen so that $\left(n^{2}+1\right) f^{\prime}(\delta)\left\|\sum_{j} y_{j}^{*} y_{j}\right\|<\varepsilon$ then all the required estimates $1^{\circ}-4^{\circ}$ hold true.

Finally, if in addition the left supports of $y_{j}$ are all under a projection $e \in A$ then $y_{j} q_{k}$ have left supports under $e$ and by [Di] the partial isometries $v_{j k}$ can be constructed so that to have left supports under $e$ as well.

Remark 2.5. Note that if $2.4 .3^{\circ}$ holds true then we also have for each $k \leq m, x \in F$ :

$$
\left\|s_{k} x s_{k}-E_{Q_{k}}\left(s_{k} x s_{k}\right)\right\|<2 \varepsilon
$$


where $Q_{k}=\sum_{i, j} \mathbb{C} e_{i j}^{k}$ and $E_{Q_{k}}(y) \stackrel{\text { def }}{=} \sum_{i, j} \tau\left(y e_{i j}^{k}\right) / \tau\left(e_{i i}^{k}\right) e_{j i}^{k}, y \in s_{k} A s_{k}$. Indeed, because if $y \in s_{k} A s_{k}$ then $\left\|E_{Q_{k}}(y)\right\| \leq\|y\|$, and thus

$$
\begin{aligned}
&\left\|s_{k} x s_{k}-E_{Q_{k}}\left(s_{k} x s_{k}\right)\right\| \leq\left\|s_{k} x s_{k}-\sum_{i, j} \tau\left(y_{i}^{*} x y_{j}\right) e_{i j}^{k}\right\| \\
&+\left\|E_{Q_{k}}\left(\sum_{i, j} \tau\left(y_{i}^{*} x y_{j}\right) e_{i j}^{k}-s_{k} x s_{k}\right)\right\| \\
& \leq 2\left\|s_{k} x s_{k}-\sum_{i, j} \tau\left(y_{i}^{*} x y_{j}\right) e_{i j}^{k}\right\| \\
& \leq 2 \varepsilon .
\end{aligned}
$$

\section{From almost invariant vector spaces to local approximation in $A$.}

We will now assume that the finite dimensional vectorspaces $\mathcal{H}_{0} \subset A$ considered in the previous section are almost invariant to the finite set $F$ and show that the matrix subalgebras $Q=Q_{k}=\sum_{i, j} \mathbb{C} e_{i j}^{k}\left(\cong \mathcal{B}\left(\mathcal{H}_{0}\right)\right) \subset A$, resulting from the local quantization of $\mathcal{H}_{0}$, will also satisfy "\|[s,F]\| small," in addition to the property " $s F s$ almost contained in $Q$," which we already had, independently on the properties of $\mathcal{H}_{0}$, where $s=1_{Q}$.

Lemma 3.1. Let $A$ be a unital $C^{*}$-algebra with a faithful trace $\tau$. Let $F=F^{*} \subset \mathcal{U}(A)$ be a selfadjoint, finite set of unitary elements in $A$ and $\varepsilon>0, \varepsilon<1 / 4$. Let $\mathcal{H}_{0}=\sum_{j=1}^{n} \mathbb{C} y_{j}$ be a finite dimensional vector subspace with $\tau\left(y_{i}^{*} y_{j}\right)=\delta_{i j}$ and $\left\{e_{i j}\right\}_{1 \leq i, j \leq n} \subset A$ a set of matrix units satisfying:

$$
\left\|s x s-\sum_{i, j} \tau\left(y_{i}^{*} x y_{j}\right) e_{i j}\right\|<\varepsilon, \quad \forall x \in F
$$

where $s=1_{Q}=\sum_{i} e_{i i}$.

If the orthogonal projection $p_{0}$ of $L^{2}(A, \tau)$ onto $\mathcal{H}_{0}$ satisfies

$$
\left\|\left[p_{0}, x\right]\right\|<\varepsilon, \quad \forall x \in F
$$


then s satisfies

$$
\|[s, x]\|<3 \varepsilon^{1 / 2}, \quad \forall x \in F .
$$

Proof. Recall that $p_{0}=\sum_{j=1}^{n} y_{j} e_{0} y_{j}^{*}$, where $e_{0}$ is the one-dimensional projection of $L^{2}(A, \varphi)$ onto $\mathbb{C} \hat{1}$, and that

$$
p_{0} x p_{0}=\sum_{i, j} \tau\left(y_{i}^{*} x y_{j}\right) y_{i} e_{0} y_{j}^{*}=\sum_{i, j} \tau\left(y_{i}^{*} x y_{j}\right) e_{i j}^{\prime}, \quad \forall x \in A \subset \mathcal{B}\left(L^{2}(A)\right)
$$

where $e_{i j}^{\prime}=y_{i} e_{0} y_{j}^{*}$ is a matrix unit for $p_{0} \mathcal{B}\left(L^{2}(A, \tau)\right) p_{0} \cong \mathcal{B}\left(\mathcal{H}_{0}\right)$.

Since $\left\|\left[p_{0}, x\right]\right\|<\varepsilon$ for $x \in F$ and since $F \subset \mathcal{U}(A)$, if we denote by $\lambda_{i j}^{x}=\tau\left(y_{i}^{*} x y_{j}\right)$ then we get

$$
\begin{aligned}
\varepsilon^{2} & >\left\|\left(1-p_{0}\right) x p_{0}\right\|^{2}=\left\|p_{0}-p_{0} x^{*} p_{0} x p_{0}\right\| \\
& =\left\|p_{0}-\left(\sum_{i, j} \lambda_{i j}^{x} e_{i j}^{\prime}\right)^{*}\left(\sum_{k, l} \lambda_{k l}^{x} e_{k l}^{\prime}\right)\right\| .
\end{aligned}
$$

But $e_{i j}^{\prime} \mapsto e_{i j}$ defines an isometric isomorphism from the algebra $\sum_{i, j} \mathbb{C} e_{i j}^{\prime}=$ $p_{0} \mathcal{B}\left(L^{2}(A, \tau)\right) p_{0}$, with support $p_{0}=\sum_{i} e_{i i}^{\prime}$, onto the algebra $Q=\sum_{i j} \mathbb{C} e_{i j}$, with support $s=\sum_{i} e_{i i}$.

Thus, from the above inequality and the hypothesis we get:

$$
\begin{aligned}
\varepsilon^{2} & >\left\|p_{0}-\left(\sum_{i, j} \lambda_{i j}^{x} e_{i j}^{\prime}\right)^{*}\left(\sum_{k, l} \lambda_{k l}^{x} e_{k l}^{\prime}\right)\right\| \\
& =\left\|s-\left(\sum_{i, j} \lambda_{i j}^{x} e_{i j}\right)^{*}\left(\sum_{k, l} \lambda_{k l}^{x} e_{k l}\right)\right\| \\
& \geq\left\|s-s x^{*} s x s\right\|-2 \varepsilon \\
& =\|(1-s) x s\|^{2}-2 \varepsilon .
\end{aligned}
$$

This finally yields:

$$
\|[s, x]\| \leq 2\left(2 \varepsilon+\varepsilon^{2}\right)^{1 / 2}<3 \varepsilon^{1 / 2}, \quad \forall x \in F
$$

(for the last estimate we used $\varepsilon<1 / 4$ ). 
The proof of Theorem 1.2 (whose statement we restate here for the reader's convenience) follows now readily:

Theorem 3.2. Let $A$ be a unital, simple $C^{*}$-algebra with sufficiently many projections (in the sense of (1.1)). Then the following conditions are equivalent:

(3.2.1) A is quasidiagonal.

(3.2.2) For any finite set $F \subset A$ and any $\varepsilon>0$ there exist a matrix subalgebra $Q \subset A, Q \neq 0$, with matrix units $\left\{e_{i j}\right\}_{1 \leq i, j \leq n}$ and support $1_{Q}=s=\sum_{i} e_{i i}$, and scalars $\lambda_{i j}^{x} \in \mathbb{C}, 1 \leq i, j \leq n, x \in F$, such that

(a) $\|[s, x]\|<\varepsilon$,

$$
\left\|s x s-\sum_{i, j} \lambda_{i j}^{x} e_{i j}\right\|<\varepsilon, \forall x \in F .
$$

Proof. (3.2.2) $\Longrightarrow(3.2 .1)$ is trivial by 1.8 in [V2] and the simplicity of $A$.

Conversely, if $A$ is quasidiagonal then it has a trace and thus, a factorial trace, say $\tau$ (faithful by the simplicity of $A$ ). Having s.m.p., $A$ has $\tau$-s.m.p. (see 2.2). By [V1], $\forall F \subset A$ finite, $\forall \varepsilon>0, \exists \mathcal{H}_{0} \subset L^{2}(A, \tau)$ such that the orthogonal projection $p_{0}$ of $L^{2}(A, \tau)$ onto $\mathcal{H}_{0}$ satisfies

$$
\left\|\left[p_{0}, x\right]\right\|<\varepsilon, \quad \forall x \in F .
$$

Since $\hat{A}$ is dense in $L^{2}(A, \tau)$ it follows that we may assume $\mathcal{H}_{0} \subset \hat{A}$. But then 2.4 and 3.1 apply, showing that $A$ satisfies (3.2.2) (a) and (b).

If instead of s.m.p. we only require $\tau$-s.m.p., for some trace $\tau$, then we need to specify this trace:

Theorem 3.3. Let $A$ be a unital, simple $C^{*}$-algebra which has $\tau$-s.m.p. for some factorial trace $\tau$ (in the sense of Definition 2.1). Then $A$ is quasidiagonal if and only if it satisfies the local finite dimensional approximation property (3.2.2).

Moreover, the following conditions are equivalent for $A$ : (3.3.1) $\forall F \subset \mathcal{U}(A)$ finite, $\forall \varepsilon>0, \exists$ a finite dimensional ${ }^{*}$-algebra $B$, with a trace $\tau_{0}$, and a completely positive unital map $\phi$ from $A$ to $B$ such that:

(a) $\left\|\phi\left(u^{*}\right) \phi(u)-1_{B}\right\|<\varepsilon, \forall u \in F$.

(b) $\left|\tau_{0} \phi(x)-\tau(x)\right|<\varepsilon, \forall x \in A,\|x\| \leq 1$.

(3.3.2) $\forall F \subset A$ finite, $\forall \varepsilon>0, \exists n \geq 1, \exists Q_{1}, \ldots, Q_{m} \subset A, Q_{k} \cong M_{n \times n}(\mathbb{C})$, $\forall k$, with supports $1_{Q_{k}}=s_{k} \in \mathcal{P}(A)$ having the same trace, $\tau\left(s_{k}\right)=\alpha, \forall k$, such that:

(a) $\left\|\left[s_{k}, x\right]\right\|<\varepsilon, \forall k, \forall x \in F$. 
(b) $\left\|s_{k} x s_{k}-E_{Q_{k}}\left(s_{k} x s_{k}\right)\right\|<\varepsilon, \forall k, \forall x$.

(c) $\left\|(m \alpha)^{-1} \sum_{k=1}^{m} s_{k}-1_{A}\right\|_{1}<\varepsilon$.

Proof. The proof of the first part is the same as the proof of 3.2.

To prove the second part, assume first that (3.3.2) holds true for some $F \subset \mathcal{U}(A)$ finite and $\varepsilon / 3$. Then $B=\oplus_{k} Q_{k}$ with the trace $\tau_{0}$ giving equal weight to each summand, $\tau_{0}\left(1_{Q_{k}}\right)=1 / m, \forall k$, and $\phi(x)=\oplus_{k} \mathrm{E}_{Q_{k}}\left(s_{k} x s_{k}\right), x \in$ $A$, are easily seen to satisfy all the conditions in (3.3.1).

Assume now that (3.3.1) holds true and want to prove that it implies (3.3.2). It is clearly suficient to do it for finite sets $F$ with $F=F^{*} \subset \mathcal{U}(A)$. By slightly modifying the trace $\tau_{0}$ we may assume all the central projections of $B$ have rational trace. Thus $B$ can be embedded trace-preservingly in a finite dimensional factor, showing that we may assume $B$ itself is a factor. By the Stinespring dilation of the completely positive map $\phi$ one then gets a Hilbert space $\mathcal{H}_{\phi}$, a representation $\pi$ of $A$ on $\mathcal{H}_{\phi}$ and a finite dimensional vector subspace $\mathcal{H}_{1} \in \mathcal{H}_{\phi}$ such that if we denote by $p_{1}$ the orthogonal projection of $\mathcal{H}_{\phi}$ onto $\mathcal{H}_{1}$ then $p_{1} \mathcal{B}\left(\mathcal{H}_{\phi}\right) p_{1}=\mathcal{B}\left(\mathcal{H}_{1}\right)$ is isomorphic to $B$ and, under this identification, we have $p_{1} \pi(x) p_{1}=\phi(x) p_{1}, \forall x \in A$. Thus

$$
\begin{aligned}
\left\|\pi(u) p_{1} \pi\left(u^{*}\right)-p_{1}\right\|^{2} & \leq 4\left\|p_{1} \pi(u) p_{1} \pi\left(u^{*}\right) p_{1}-p_{1}\right\| \\
& =4\left\|\left(\phi(u) \phi\left(u^{*}\right)-1_{B}\right) p_{1}\right\|<4 \varepsilon .
\end{aligned}
$$

Moreover if $\operatorname{Tr}$ denotes the trace on $\mathcal{B}\left(\mathcal{H}_{\phi}\right)$ then

$$
\operatorname{Tr}\left(\pi(x) p_{1}\right) / \operatorname{Tr}\left(p_{1}\right)=\operatorname{Tr}\left(p_{1} \pi(x) p_{1}\right) / \operatorname{Tr}\left(p_{1}\right)=\tau_{0}(\phi(x)) .
$$

Denote by $\mathcal{H}^{\prime} \subset \mathcal{H}_{\phi}$ the subspace of all vectors $\zeta \in \mathcal{H}_{\phi}$ for which the positive form $\langle\pi(x) \zeta, \zeta\rangle$ on $A$ is given by an elemnt in $\mathrm{L}^{1}(A, \tau)=\mathrm{L}^{1}(M, \tau)$, where $M$ denotes as usual the weak closure (or the bicommutant) of $A$ in $\mathcal{B}\left(\mathrm{L}^{2}(A, \tau)\right)$. Thus, $\mathcal{H}^{\prime}$ is a closed vector subspace of $\mathcal{H}_{\phi}$, which is clearly invariant to $\pi(x), \forall x \in A$. Thus, if $p^{\prime}$ denotes the corresponding orthoganal projection onto $\mathcal{H}^{\prime}$, then $p^{\prime}$ commutes with $\pi(A)$ and we have

$$
\left\|\pi(u) p^{\prime} p_{1} p^{\prime} \pi\left(u^{*}\right)-p^{\prime} p_{1} p^{\prime}\right\|<2 \varepsilon^{1 / 2}, \forall u \in F .
$$

Since $\operatorname{Tr}\left(\pi(x) p_{1}\right)=\operatorname{Tr}\left(\pi(x) p^{\prime} p_{1} p^{\prime}\right)+\operatorname{Tr}\left(\pi(x)\left(1-p^{\prime}\right) p_{1}\left(1-p^{\prime}\right)\right)$, by using the fact that for $\varphi \in \mathrm{L}^{1}(A, \tau)$ and $\psi=\langle\pi() \zeta, \zeta\rangle$, with $\zeta \in\left(1-p^{\prime}\right) \mathcal{H}_{\phi}$, we have $\|\varphi-\psi\|=\|\varphi\|+\|\psi\|$, it follows that:

$$
\begin{aligned}
\varepsilon>\left\|\tau_{0} \varphi-\tau\right\|= & \left\|\operatorname{Tr}\left(\pi() p^{\prime} p_{1} p^{\prime}\right) / \operatorname{Tr}\left(p_{1}\right)-\tau()\right\| \\
& +\left\|\operatorname{Tr}\left(\pi()\left(1-p^{\prime}\right) p_{1}\left(1-p^{\prime}\right)\right) / \operatorname{Tr}\left(p_{1}\right)\right\| .
\end{aligned}
$$

In particular, it follows that

$$
\left\|\operatorname{Tr}\left(\pi() p^{\prime} p_{1} p^{\prime}\right) / \operatorname{Tr}\left(p_{1}\right)-\tau()\right\|<\varepsilon
$$




$$
\left|\operatorname{Tr}\left(p^{\prime} p_{1} p^{\prime}\right)-\operatorname{Tr}\left(p_{1}\right)\right|<\varepsilon \operatorname{Tr} p_{1} .
$$

From all the above, by using [Di] and [C1], it follows that there exists a spectral projection $p_{0}$ of $p^{\prime} p_{1} p^{\prime}$ such that:

$$
\begin{gathered}
\left\|\pi(u) p_{0} \pi\left(u^{*}\right)-p_{0}\right\|<f(\varepsilon), \forall u \in F, \\
\left\|\operatorname{Tr}\left(\pi() p_{0}\right) / \operatorname{Tr}\left(p_{1}\right)-\tau\right\|<f(\varepsilon), \\
\left|\operatorname{Tr}\left(p_{0}\right)-\operatorname{Tr}\left(p_{1}\right)\right|<f(\varepsilon) \operatorname{Tr} p_{1},
\end{gathered}
$$

where $f(\varepsilon)$ is a constant depending on $\varepsilon$, tending to 0 when $\varepsilon$ tends to 0 . Moreover, if $\mathcal{H}=\overline{\mathrm{sp}} A p_{0} \mathcal{H}^{\prime}$, then $\mathcal{H}$ is invariant to $\pi(A)$ and Range $p_{0} \subset \mathcal{H}$, and so we may "cut" with the projection on $\mathcal{H}$ and still have the above estimates. Altogether, we may assume $\pi$ is a representation of $A$ on $\mathcal{H}$. Note that when regarded this way $\pi$ extends to a normal representation of $M$ and that $\pi(M)$ has finite coupling constant $c \leq \operatorname{Tr} p_{0}$. Moreover, by taking if necessary a direct sum of $\mathcal{H}$ with with an appropriate normal $M$ Hilbert module, we may assume the coupling constant $c$ is an integer, $c=k$. Thus, $\mathcal{H}=\mathrm{L}^{2}\left(e M_{1}, \tau\right)$, where $M_{1}=\mathrm{M}_{k \times k}(M)$ and $e \in \mathrm{M}_{k \times k}(\mathbb{C}) \subset M_{1}$ is a projection of trace $1 / k$, and where the scalar product in $\mathcal{H}$ is given by $\tau\left(x_{1} y_{1}^{*}\right)$, for $x_{1}, y_{1} \in e M_{1}, \tau$ being the normalized trace on $M \simeq M e=e M_{1} e$. Since $e \hat{A}_{1}$ is dense in $\mathrm{L}^{2}\left(e M_{1}, \tau\right)$ in the norm \|\|$_{2}$, where $A_{1}=\mathrm{M}_{k \times k}(A) \subset$ $\mathrm{M}_{k \times k}(M)=M_{1}$, it follows that we may assume $p_{0} \mathcal{H} \subset e \hat{A}_{1}$. Let $p_{0} \mathcal{H}=$ $\sum_{j=1}^{m} \mathbb{C} \hat{y}_{j}$, with $y_{j} \in e A_{1}, \tau\left(y_{i} y_{j}^{*}\right)=\delta_{i j}$. The condition $\| \operatorname{Tr}\left(\pi() p_{0}\right) / \operatorname{Tr} p_{0}-$ $\tau() \|<2 f(\varepsilon)$ and the assumption (3.3.1) then give:

$$
\left|m^{-1} \tau\left(x \sum_{j} y_{j} y_{j}^{*}\right) /-\tau(x)\right|<f^{\prime}(\varepsilon), \forall x \in e A_{1} e=A e,\|x\| \leq 1,
$$

which implies that in the norm $\|x\|_{1, \tau}=\tau(|x|)$, for $x \in M e$, we have

$$
\left\|m^{-1} \sum_{j} y_{j} y_{j}^{*}-e\right\|_{1}<f^{\prime}(\varepsilon) \text {. }
$$

Thus, if $\tau_{1}$ denotes the normalized trace on $A_{1} \subset M_{1}$ then $\tau_{1}\left(y_{j}^{*} y_{i}\right)=$ $k^{-1} \delta_{i j}=\tau_{1}\left(y_{i} y_{j}^{*}\right)$ and also

$$
\left\|m^{-1} \sum_{j} y_{j} y_{j}^{*}-e\right\|_{1, \tau_{1}}<f^{\prime}(\varepsilon) \tau_{1}(e)
$$

with $f^{\prime}(\varepsilon)$ tending to 0 when $\varepsilon$ tends to 0 . Property (3.3.2) follows now imediately, by taking into account that if $A$ has $\tau$-s.m.p. then $A_{1}$ has $\tau_{1}$ s.m.p. and then applying 2.4 (including its last part) to $A_{1}$ and the $\tau_{1}$ orthonormal set of elements $k^{1 / 2} y_{j} \in e A_{1} \subset A_{1}$. 
Remarks 3.4. $1^{\circ}$. Note that if a unital $\mathrm{C}^{*}$-algebra with unique trace is embeddable in a unital AF algebra with a faithful trace then it does satisfy condition (3.3.1) and so, by the above theorem, it satisfies (3.3.2) as well. A large class of $\mathrm{AF}$-embeddable $\mathrm{C}^{*}$ algebras have been found in $[\mathbf{P i}]$. Most of these algebras are easily seen to have s.m.p., or $\tau$-s.m.p.

$2^{\circ}$. If a separable $\mathrm{C}^{*}$ algebra $A$ is simple, has $\tau$-s.m.p. for some factorial trace $\tau$ and satisfies (3.3.2) then $M=A^{\prime \prime} \subset \mathcal{B}\left(\mathrm{L}^{2}(A, \tau)\right)$ is a hyperfinite factor. To see this, let $\left\{x_{j}\right\}_{j}$ be a sequence of elements dense in the unit ball of $A$ in the norm topology, and let $\left\{Q_{k}^{n}\right\}_{1 \leq k \leq m_{n}}$ be matrix subalgebras of $A$, of supports $1_{Q_{k}^{n}}=s_{k}^{n} \in \mathcal{P}(A)$, which satisfy (3.3.2) for $F_{n}=\left\{x_{j}\right\}_{1 \leq j \leq n}$, $\varepsilon=1 / n$. Then let $\mathcal{U}_{k}^{n}$ denote the unitary group of $Q_{k}^{n}$ and $\mathrm{d} u$ its Haar measure. For $T \in \mathcal{B}\left(\mathrm{L}^{2}(A, \tau)\right)$ we put:

$$
\varphi(T)=\lim _{n \rightarrow \omega}\left(\sum_{k}\left\langle\left(\int_{\mathcal{U}_{k}^{n}} u T u^{*} \mathrm{~d} u\right) \hat{s}_{k}^{n}, \hat{s}_{k}^{n}\right\rangle / \sum_{k} \tau\left(s_{k}^{n}\right)\right) .
$$

By using [C2], it is then easy to see that $\varphi$ this way defined is a hypertrace for $M=A^{\prime \prime} \subset \mathcal{B}\left(\mathrm{L}^{2}(A, \tau)\right)$, so $M$ follows hyperfinite by Connes' fundamental theorem $([\mathbf{C} \mathbf{1}])$. It is not clear whether or not (3.3.2) implies that in fact all the factor representations of $A$ are hyperfinite, so that quasidiagonality, in its stronger (3.3.1)-version, would actually imply nuclearity (see also the remarks at the end of next section). Also, we do not know whether or not condition (3.2.2) is actually suficient for $M$ to be hyperfinite. This latter problem seems to be easier to settle, though.

\section{Some remarks.}

Note that if $A=M$ is a type $\mathrm{II}_{1}$ factor then the Hilbert-Schmidt-norm version of the local finite dimensional approximation condition (3.1.1) in the definition of quasidiagonality is just Connes' Følner type condition ([C1]): $\forall F \subset M$ finite, $\forall \varepsilon>0, \exists \mathcal{H}_{0} \subset L^{2}(M, \tau)$ finite dimensional vector space, such that

$$
\left\|\left[\operatorname{proj}_{\mathcal{H}_{0}}, x\right]\right\|_{\mathrm{HS}}<\varepsilon\left\|\operatorname{proj}_{\mathcal{H}_{0}}\right\|_{\mathrm{HS}}, \forall x \in F
$$

where $\|X\|_{\mathrm{HS}}=\operatorname{Tr}\left(X^{*} X\right)^{1 / 2}$ and $\operatorname{Tr}$ is the trace on $\mathcal{B}\left(L^{2}(M, \tau)\right)$.

If $\mathcal{H}_{0}, F=F^{*} \subset \mathcal{U}(M), \varepsilon$ are such that (4.1) is satisfied and if $Q \subset M$ is the $n$ by $n$ matrix algebra with support $s \in \mathcal{P}(M)$ associated with $\mathcal{H}_{0}, F, \varepsilon$ 
by local quantization, as in 2.4, then with the notations of 3.1 we have

$$
\begin{aligned}
n \varepsilon^{2} & =\varepsilon^{2} \operatorname{Tr} p_{0}>\left\|\left(1-p_{0}\right) x p_{0}\right\|_{\mathrm{HS}}^{2} \\
& =\operatorname{Tr}\left(p_{0}-p_{0} x^{*} p_{0} x p_{0}\right)=\operatorname{Tr}\left(p_{0}-\left(\sum_{i, j} \lambda_{i j}^{x} e_{i j}^{\prime}\right)^{*}\left(\sum_{k l} \lambda_{k l}^{x} e_{k l}^{\prime}\right)\right) \\
& =\frac{n}{\tau(s)} \tau\left(s-\left(\sum_{i, j} \lambda_{i j}^{x} e_{i j}\right)^{*}\left(\sum_{k l} \lambda_{k l}^{x} e_{k l}\right)\right) \\
& \geq \frac{n}{\tau(s)}\left(\tau\left(s-s x^{*} s x s\right)-2 \varepsilon \tau(s)\right) \\
& =\frac{n}{\tau(s)}\left(\|(1-s) x s\|_{2}^{2}-2 \varepsilon \tau(s)\right)
\end{aligned}
$$

in which we used that the isomorphism $e_{i j}^{\prime} \mapsto e_{i j}$ from $p_{0} \mathcal{B}\left(L^{2}(M, \tau)\right) p_{0}=$ $\sum_{i, j} \mathbb{C} e_{i j}^{\prime}$ onto $Q=\sum_{i, j} \mathbb{C} e_{i j}$ takes the normalized trace $(1 / n) \operatorname{Tr}$ onto the normalized trace $(1 / \tau(s)) \tau$. Thus we get for $x \in F=F^{*}$ :

$$
\begin{aligned}
\|[s, x]\|_{2}^{2} & \leq 2\|(1-s) x s\|_{2}^{2}+2\|s x(1-s)\|_{2}^{2} \\
& =2\left(\|(1-s) x s\|_{2}^{2}+\left\|(1-s) x^{*} s\right\|_{2}^{2}\right) \\
& \leq 2\left(\varepsilon^{2}+2 \varepsilon\right) \tau(s)
\end{aligned}
$$

which for $\varepsilon<1 / 4$ gives:

$$
\|[s, x]\|_{2}<3 \varepsilon^{1 / 2}\|s\|_{2}, \forall x \in F .
$$

This estimate adds to the Estimate 2.4 that we already had:

$$
\left\|s x s-\sum_{i, j} \lambda_{i j}^{x} e_{i j}\right\|<\varepsilon, \forall x \in F
$$

and which trivially implies:

$$
\left\|s x s-E_{Q}(s x s)\right\|_{2}<\varepsilon\|s\|_{2}, \forall x \in F .
$$

From this local finite dimensional approximation property in the norm $\|\quad\|_{2}$, inside $M$, one can then easily obtain a global finite dimensional approximation (inside $M$ ), by using a maximality argument. Altogether this proves that Connes' Følner type condition on $M$ implies $M$ is AFD (approximately finite dimensional), thus giving an alternative proof to Connes' uniqueness of the injective type $\mathrm{II}_{1}$ factor, along the scheme: " $M$ injective $\Leftrightarrow M$ has a hypertrace $\Leftrightarrow M$ satisfies the Condition (4.1) $\Rightarrow M$ fas the 
local AFD Property (4.2) $\Rightarrow M$ is AFD $\Rightarrow M$ is isomorphic to the hyperfinite type $\mathrm{II}_{1}$ factor $R$ ", the last implication being Murray-von Neumann's theorem (see [Po1] for details of this).

As we have seen, in case $\mathcal{H}_{0}$ are almost invariant in the uniform norm, i.e., satisfy the stronger condition (3.1.1), then one obtains the local finite dimensional approximation (inside the algebra) in uniform norm, which clearly implies (4.2). But we can no longer "glue" the algebras $(Q, s)$ by a maximality argument, to obtain a global approximation, in the uniform norm, by finite dimensional subalgebras, as one can do for the norm \|\|$_{2}$.

Still, the last part of Theorem 3.3 and the remark following it show that for certain quasidiagonal $\mathrm{C}^{*}$-algebras a weak type of (almost) global finite dimensional approximation can be obtained. This fact might be useful for trying to prove (or disprove) that a unital quasidiagonal $\mathrm{C}^{*}$-algebra with unique trace and sufficiently many projections (or even real rank zero) is necessarily nuclear (note that the exemples of non-nuclear $\mathrm{C}^{*}$-subalgebras in UHF algebras in $[\mathbf{B l}]$ are not simple). Moreover, related to this same problem note that, under suitable conditions, in Theorem 2.4 one can choose the projections $q$ in a given $\mathrm{C}^{*}$-subalgebra of $A$. This fact may also be useful when trying to prove that a nuclear quasidiagonal $\mathrm{C}^{*}$-algebra $A$, with a unique trace, real rank zero and trivial $\mathrm{K}_{1}$ group is necessarily AF. Giving an abstract characterizations of AF algebras was proposed as a problem in $[\mathbf{E}]$ and would now be a crucial step towards solving Elliott's conjecture ([Ell] $)$. Indeed, both in the "quasidiagonality (+finiteness+simplicity+r.r.0) implies nuclearity problem" and the "AF algebra problem" one possible strategy would be to say construct certain maximal nuclear C-* algebras $B$ of $A$ (maximal AF for the second problem) and then showing that $B$ must equal A.

\section{References}

[B] J. Bellissard, C-* algebras in solid state physics, in "Operator algebras and applications", (1988), D. Evans and M. Takesaki Eds., Cambridge University Press.

[BPo] J. Bellissard and S. Popa, unpublished.

[Bl] B. Blackadar, Nonnuclear subalgebras of $C^{*}$ * algebras, J. Operator Theory, 14(2) (1985), 347-350.

[BlK] B. Blackadar and E. Kirchberg, Inner quasidiagonality and strong NF algebras, preprint 1996.

[BrP] L. Brown and G. Pedersen, C-* algebras of real rank zero, 1989.

[C1] A. Connes, Classification of injective factors, Ann. Math., 104 (1976), 73-115.

[C2] Compact metric spaces, Fredholm modules and hyperfiniteness, Ergod. Th. \& Dynam. Sys., 9 (1989), 207-220. 


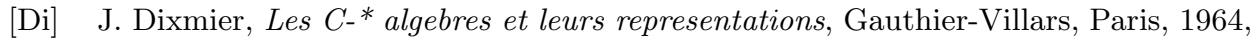
1968.

[E] E. Effros, On the structure theory of $C$-* algebras: Some old and new problems, in "Proceedings of Symposia in Pure Mathematics", Vol. 38, (1982), 19-34.

[Ell] G. Elliott, The classification problem for amenable $C_{-}{ }^{*}$ algebras, in "Proceedings of the International Congress of Mathematicians", Zurich 1994, Birkhauser, Berlin, 922-932.

[Pi] M. Pimsner, Embedding some transformation group $C$-* algebras into AF-algebras, Ergodic Th. and Dynamical Syst., 3 (1983), 613-626.

[Po1] S. Popa, On amenable factors of type $\mathrm{II}_{1}$, in "Operator Algebras and Applications," London Math. Lect. Ser., 136, 173-184, Cambridge University Press, 1988.

[Po2] - Free independent sequences in type $\mathrm{II}_{1}$ factors, Asterisque, 172 (1995), 187-202.

[R] J. Renault, A groupoid approach to $C$ - $^{*}$ algebras, Lecture Notes in Mathematics, 793, Springer Verlag, Berlin, 1980.

[V1] D. Voiculescu, A non-commutative Weyl-von Neumann type theorem, Rev. Roumaine Math. Pures Appl., 21 (1976), 97-113.

[V2] Around quasi-diagonal operators, Integral Equations Operator Theory, 17 (1993), 137-149.

[V3] Circular and semicircular systems and free product factors, in "Operator Algebras, Unitary Representations, Enveloping Algebras", Progress in Mathematics, Vol. 92, Birkhauser, 1990, 45-60.

Received December 13, 1996.

University OF CALIFORNIA

LOS ANGELES, CA 90095-1555

E-mail address: popa@math.ucla.edu 